\title{
Topological conjugacy of cascades generated by gradient flows on the two-dimensional sphere
}

\author{
by ANDRZEJ BieleCKI (Kraków)
}

\begin{abstract}
This article presents a theorem about the topological conjugacy of a gradient dynamical system with a constant time step and the cascade generated by its Euler method. It is shown that on the two-dimensional sphere $\mathcal{S}^{2}$ the gradient dynamical flow is, under some natural assumptions, correctly reproduced by the Euler method for a sufficiently small time step. This means that the time-map of the induced dynamical system is globally topologically conjugate to the discrete dynamical system obtained via the Euler method.
\end{abstract}

1. Introduction. In recent years several papers have been devoted to studying the qualitative properties of discrete-time dynamical systems obtained via discretization methods. The basic question is whether the qualitative properties of continuous-time systems are preserved under discretization. Various concepts of differentiable dynamics were investigated. Results on stability and attraction properties ([KL]), the saddle-point structure about equilibria ([AD], [Bey1], [Bey2]), invariant manifolds ([BL], [Fec1]), averagings $([\mathrm{Fec} 2])$ and algebraic-topological invariants $([\mathrm{MR}])$ can be mentioned as examples. A number of applications have been studied as well ([Gar4]). The investigations are concerned with both local (see, for instance, [Gar1], [Fec3]) and global conjugacy ([Gar2], [Gar3], [Gar5]).

This paper is devoted to the problem of topological conjugacy between the discretization of a gradient dynamical system and the cascade generated by its Euler method. Similar problems have been solved in recent years for numerical methods of order greater than one (see [Gar2], [Li]).

2. Topological conjugacy of gradient cascades. As mentioned above we consider a gradient differential equation and its Euler method. The timemap of the induced solution is compared to the cascade obtained via the

2000 Mathematics Subject Classification: 34C35, 34A50.

Key words and phrases: topological conjugacy, gradient dynamical system, Euler method. 
Euler method. We show that on the two-dimensional sphere $\mathcal{S}^{2}$ a gradient dynamical system is, under some natural assumptions, correctly reproduced by the Euler method for a sufficiently small time step. This means that the time-map of the induced dynamical system is globally topologically conjugate to the discrete dynamical system obtained via the Euler method. This fact can be expressed as follows.

THEOREM 2.1. Let $\mathcal{S}^{2}$ be the unit sphere in $\mathbb{R}^{3}$ and let

$$
\phi: \mathcal{S}^{2} \times \mathbb{R} \rightarrow \mathcal{S}^{2}
$$

be the dynamical system generated by a differential gradient equation

$$
\dot{x}=-\operatorname{grad} E(x),
$$

where $E \in \mathcal{C}^{2}\left(\mathcal{S}^{2}, \mathbb{R}\right)$, having a finite number of singularities, all hyperbolic. Let, furthermore, the dynamical system $\phi$ have no saddle-saddle connections. Moreover, let $\phi_{h}: \mathcal{S}^{2} \rightarrow \mathcal{S}^{2}$ be the discretization of $\phi$, i.e. $\phi_{h}(x):=$ $\phi(x, h)$, and let $\psi_{h}: \mathcal{S}^{2} \rightarrow \mathcal{S}^{2}$ be generated by the Euler method for (1). Then, for sufficiently small $h>0$, there exists a homeomorphism $\alpha=\alpha_{h}$ : $\mathcal{S}^{2} \rightarrow \mathcal{S}^{2}$ globally conjugating the cascades generated by $\phi_{h}$ and $\psi_{h}$, i.e.

$$
\phi_{h} \circ \alpha=\alpha \circ \psi_{h} .
$$

REMARKS. 1. Axiom A and the strong transversality condition are known to be equivalent to the structural stability of a dynamical system (see [PM], p. 171, and [Man]). On the other hand, for gradient dynamical systems, Axiom A implies that the system has only a finite number of singularities, all hyperbolic, whereas the strong transversality condition implies that the system has no saddle-saddle connections. Thus, the structural stability of a dynamical system $\left(\mathcal{S}^{2}, \phi\right)$ implies the assumptions of Theorem 2.1. Moreover, the set of structurally stable systems is open and dense in the space of gradient dynamical systems (see $[\mathrm{PM}]$, p. 116).

2. A dynamical system generated by the equation (1), having only a finite number of singularities, all hyperbolic, without saddle-saddle connections is called a gradient Morse-Smale system.

3. Estimation of the Euler method on $\mathcal{S}^{n}$. Let $n_{0} \in \mathbb{N}$ and let $a=n_{0} h$ denote the length of the time interval on which the error $e_{n}:=$ $\varrho_{\mathcal{M}}\left(\phi_{h}^{n_{0}}(x), \psi_{h}^{n_{0}}(x)\right)$ is estimated $\left(\varrho_{\mathcal{M}}\right.$ denotes the Riemannian metric on $\mathcal{M})$. We will show that on the $n$-dimensional sphere

$$
e_{n}<\xi(a) h
$$

where, for a given problem, $\xi(a)$ is a constant value which only depends on $a$.

Consider the problem

$$
\dot{x}=f(x), \quad x(0)=x_{0}, \quad 0 \leq t \leq a,
$$


on a compact manifold $\mathcal{M}$, where $f$ is a vector field on $\mathcal{M}$. The Euler iterative rule for the problem (4) is of the form

$$
x_{n}=\exp _{x_{n}}\left(-h f\left(x_{n-1}\right)\right),
$$

where $f\left(x_{n-1}\right)$ is a vector of the tangent space $T_{x_{n-1}} \mathcal{M}$.

The Euler method in $\mathbb{R}^{n}$ is a first order numerical method ([Kru], p. 31). For $x, y \in \mathcal{M}$ in the domain of a chart $\vartheta$ of a manifold $\mathcal{M}$, we have

$$
m_{1} \varrho(\vartheta(x), \vartheta(y)) \leq \varrho_{\mathcal{M}}(x, y) \leq m_{2} \varrho(\vartheta(x), \vartheta(y)),
$$

where $m_{1}, m_{2}$ are constant for a given chart $\vartheta$ (see [Rob], p. 453, formula $(2.2))$; $\varrho$ is the euclidian metric on $\mathbb{R}^{n}$. By (6), the Euler method on a compact manifold is also a first order method.

The error in a single step of a numerical method is defined by

$$
r(x, h):=\varrho_{\mathcal{M}}\left(\psi_{h}(x), \phi_{h}(x)\right)
$$

and is a continuous function of $x$ for a constant $h$. Therefore, on a compact manifold, it reaches its maximum. Set

$$
r(h):=\max _{x \in \mathcal{M}} r(x, h) .
$$

Let us estimate the error of the Euler method for a gradient equation on the sphere $\mathcal{S}^{n}$ in $\mathbb{R}^{n+1}$. By the assumptions of Theorem 2.1 a gradient dynamical system on a compact manifold has at least one attracting singularity. Change coordinates in $\mathbb{R}^{n+1}$ so that this fixed point is the south pole of the sphere. The sphere can be covered by two charts $\vartheta_{1}, \vartheta_{2}$ with

$$
\vartheta_{1}: \mathcal{S}^{n} \backslash p_{\text {north }} \rightarrow \mathbb{R}^{n}, \quad \vartheta_{2}: \mathcal{S}^{n} \backslash p_{\text {south }} \rightarrow \mathbb{R}^{n},
$$

where $p_{\text {north }}, p_{\text {south }}$ are the poles of the sphere.

If the south pole is the starting point of the Euler method then

$$
\varrho_{\mathcal{M}}\left(\phi_{h}^{n}(x), \psi_{h}^{n}(x)\right)=0
$$

for each $n \in \mathbb{N}$ as the gradient is zero at the south pole. In this case the error is zero.

In the other case, if the starting point $x$ is different from $p_{\text {south }}$ then $\phi_{h}^{n}(x) \neq p_{\text {south }}$ for each $n \in \mathbb{N}$, because $\psi_{h}$ is invertible for $h$ sufficiently small. Thus, for each $n \leq n_{0}$, both $\phi_{h}^{n}(x)$ and $\psi_{h}^{n}(x)$ lie in the domain of the chart $\vartheta_{2}$. Therefore, the point $x$ and the systems $\phi_{h}$ and $\psi_{h}$ can be transformed into $\mathbb{R}^{n}$ in order to perform the iterations $\widetilde{\psi}_{h}^{n_{0}}\left(\vartheta_{2}(x)\right)$ and $\widetilde{\phi}_{h}^{n_{0}}\left(\vartheta_{2}(x)\right)$. Afterwards, we return to the sphere via $\vartheta_{2}^{-1}$. The dynamical systems $\widetilde{\psi}_{h}$ and $\widetilde{\phi}_{h}$ are the systems $\psi_{h}$ and $\phi_{h}$ transformed from the sphere to $\mathbb{R}^{n}$ by the maps of the atlas. Notice that if $\psi_{h}$ is generated by the Euler method of the gradient equation generating $\phi_{h}$ on the sphere then $\widetilde{\psi}_{h}$ is generated by the Euler method of the equation generating $\widetilde{\phi}_{h}$ in $\mathbb{R}^{n}$ because the atlas preserves the differential structure. 
The error estimate with step $h$ for the equation

$$
\dot{x}=f(x),
$$

where $f$ is lipschitzian with a constant $L$ in $\mathbb{R}^{n}$, is given by the formula (see [Kru], p. 32, formula 1.22)

$$
e_{n} \leq\left(e_{0}+\frac{r h}{L}\right) e^{L a},
$$

where $a=n_{0} h$ is the time interval on which the solution is considered, $r h^{2}$ is the maximal error in a single step and $e_{0}$ is the initial error. Since the right side of (1), being a differentiable map on a compact space, is a lipschitzian map, so is the right side of the equation transformed to $\mathbb{R}^{n}$, by (6). Therefore, the estimate (8) can be applied to the systems $\widetilde{\psi}_{h}$ and $\widetilde{\phi}_{h}$. The flow and its Euler method start from the same point, thus $e_{0}=0$ and

$$
\varrho\left(\widetilde{\psi}_{h}^{n_{0}}\left(\vartheta_{2}(x)\right), \widetilde{\phi}_{h}^{n_{0}}\left(\vartheta_{2}(x)\right)\right) \leq \frac{r h}{L} e^{L a} .
$$

By (6) we get the following estimate on $\mathcal{S}^{n}$ :

$$
\varrho_{\mathcal{M}}\left(\psi_{h}^{n_{0}}(x), \phi_{h}^{n_{0}}(x)\right) \leq m_{2} \frac{r h}{L} e^{L a},
$$

which is of the same form as in $\mathbb{R}^{n}$. Thus we have obtained (3).

4. Lemmas. One of the key points in the proof of Theorem 2.1 is a construction of proper homeomorphisms conjugating the cascades $\phi_{h}$ and $\psi_{h}$ in a neighbourhood of an attracting singularity (see Lemma 4.6). This construction is based on the following geometric lemma.

LEMMA 4.1. Let $\gamma_{1}, \gamma_{2}, \delta_{1}, \delta_{2}$ be curves in $\mathbb{R}^{2}$, each parametrized by $\tau \in$ $[0,1]$, homeomorphic to a line segment and such that $\gamma_{1}(0)=\delta_{1}(0), \gamma_{1}(1)=$ $\delta_{2}(0), \gamma_{2}(0)=\delta_{1}(1)$ and $\gamma_{2}(1)=\delta_{2}(1)$. Assume that their union is the boundary of a simply connected domain $\mathcal{D}$. Then there exists a homeomorphism $\Lambda: \mathcal{D} \rightarrow[0,1]^{2}$ such that $\Lambda\left(\gamma_{1}\right)=[0,1] \times\{1\}, \Lambda\left(\gamma_{2}\right)=[0,1] \times$ $\{0\}, \Lambda\left(\delta_{1}\right)=\{1\} \times[0,1], \Lambda\left(\delta_{2}\right)=\{0\} \times[0,1]$.

The proof of this well known fact is omitted. It can be found in [Bie].

A great number of theorems concerning the topological conjugacy near hyperbolic singularities are known. We will need the following theorem (see [Bey], [Gar2], [Gar3]).

THEOREM 4.2. For each equilibrium point $x_{0}$ of the cascade $\phi_{h}$ and for sufficiently small $h$ there exists an equilibrium point $x_{h}$ of the cascade $\psi_{h}$, a neighbourhood $V_{x_{0}}$ of $x_{0}$, and a homeomorphism $\alpha_{h}: V_{x_{0}} \rightarrow \alpha_{h}\left(V_{x_{0}}\right)$ such that $\alpha_{h}\left(x_{0}\right)=x_{h}$ and $\left(\alpha_{h} \circ \phi_{h}\right)(x)=\left(\psi_{h} \circ \alpha_{h}\right)(x)$, whenever $x$ and $\phi_{h}(x)$ are in $V_{x_{0}}$. 
In our case, since $x_{h}$ is an equilibrium point of $\psi_{h}$ iff $\operatorname{grad} E\left(x_{h}\right)=0$, we have $x_{h}=x_{0}$ for every $h>0$.

Throughout this section $\mathcal{M}$ denotes a compact differentiable manifold of dimension greater than one, $\phi$ denotes a Morse-Smale gradient system on $\mathcal{M}$, and $\phi_{h}$ and $\psi_{h}$ are the cascades defined as in Theorem 2.1. Let, furthermore,

$$
g_{c_{i}}: V_{c_{i}} \rightarrow V_{c_{i}}^{*}
$$

be a homeomorphism locally conjugating $\phi_{h}$ and $\psi_{h}$ in the neighbourhood $V_{c_{i}}$ of the singularity $c_{i}$ and $V_{c_{i}}^{*}=g\left(V_{c_{i}}\right)$.

LEMMA 4.3. Let $c_{i}$ be an attracting or saddle singularity. Then there exists $\overline{\bar{r}}_{i}>E\left(c_{i}\right)$ such that for every $x \neq c_{i}$ in $W_{\phi}^{\mathrm{s}}\left(c_{i}\right) \cap V_{c_{i}}$ the following implication holds: if $E(x) \leq \overline{\bar{r}}_{i}$ then there exists $t>0$ such that

$$
E(\phi(x,-t))>\overline{\bar{r}}_{i} \text { and } \phi(x,-t) \in W_{\phi}^{\mathrm{s}}\left(c_{i}\right) \cap V_{i} .
$$

Furthermore the sets

$$
\begin{aligned}
& K_{c_{i}, 0}:=\left\{x \in W_{\phi}^{\mathrm{s}}\left(c_{i}\right): E(x) \leq \overline{\bar{r}}_{i}\right\}, \\
& K_{c_{i}, 1}:=\left\{x \in W_{\phi}^{\mathrm{s}}\left(c_{i}\right): E(x) \leq \bar{r}_{i}\right\}, \quad \text { where } E\left(c_{i}\right)<\bar{r}_{i}<\overline{\bar{r}}_{i},
\end{aligned}
$$

are nonempty.

Proof. The point $c_{i}$ is attracting on the stable manifold $W_{\phi}^{\mathrm{s}}\left(c_{i}\right)$. Choose

$$
R<\min \left\{\varrho_{\mathcal{M}}\left(c_{i}, c_{j}\right): i \neq j, c_{j} \text { is a fixed point }\right\} .
$$

Let $B_{\text {rel }}\left(c_{i}, R\right)$ be the closed ball and $S_{\text {rel }}\left(c_{i}, R\right)$ the sphere in $W_{\phi}^{\mathrm{s}}\left(c_{i}\right)$. By compactness there exists $x_{0} \in S_{\text {rel }}\left(c_{i}, R\right)$ such that

$$
E\left(x_{0}\right)=\inf \left\{E(x): x \in S_{\text {rel }}\left(c_{i}, R\right)\right\}=: e_{i} .
$$

The following proposition is necessary to complete the proof of Lemma 4.3. Its proof follows simply from the Morse lemma and therefore is omitted (it can be found in [Bie]).

Proposition 4.4. Let $e_{i}>r^{*}>E\left(c_{i}\right)$. Then the set

$$
B_{\text {lev }}\left(c_{i}, r^{*}\right):=\left\{x \in W_{\phi, c_{i}}^{\mathrm{s}}: E(x) \leq r^{*}\right\}
$$

and the ball $B_{\mathrm{rel}}\left(c_{i}, R\right)$ are homeomorphic.

Since the potential $E$ decreases along each orbit, every $\overline{\bar{r}}_{i}<r^{*}$ (where $r^{*}$ is defined in Corollary 4.4) satisfies the implication in Lemma 4.3. That implies that the sets $K_{c_{i}, m}, m=0,1$, are nonempty.

Lemma 4.5. The sets $K_{c_{i}, m}, m=1,2$, have the following properties:

(i) $c_{i} \in \operatorname{int}_{\mathrm{rel}} K_{c_{i}, m}$,

(ii) $\partial_{\text {rel }} K_{c_{i}, 0}=\left\{x \in W_{\phi, c_{i}}^{\mathrm{s}}: E(x)=\overline{\bar{r}}\right\}$ and $\partial_{\mathrm{rel}} K_{c_{i}, 1}=\left\{x \in W_{\phi, c_{i}}^{\mathrm{s}}\right.$ : $E(x)=\bar{r}\}$,

(iii) $\{\phi(x, t): t>0\} \subset K_{c_{i}, m}$ for every $x \in K_{c_{i}, m}$, 
(iv) $K_{c_{i}, m}$ is arcwise connected,

(v) $\inf \left\{\varrho_{\mathcal{M}}(x, y): x \in K_{c_{i}, 1}, y \in K_{c_{i}, 0}\right\}>0$.

The boundaries and interiors are considered in the relative topology of $W_{\phi, c_{i}}^{\mathrm{s}}$ and therefore are marked by the subscript "rel".

Proof. (i) This is clear since $K_{c_{i}, m}$ is homeomorphic to a ball $B_{\text {rel }}\left(c_{i}, r\right)$.

(ii) Let $x_{0} \in \partial_{\text {rel }} K_{c_{i}, 0}$. Then, for every neighbourhood $V_{x_{0}}$ of $x_{0}$,

$V_{x_{0}} \cap\left\{x \in W_{\phi, c_{i}}^{\mathrm{s}}: E(x) \leq \overline{\bar{r}}\right\} \neq \emptyset$ and $V_{x_{0}} \cap\left\{x \in W_{\phi, c_{i}}^{\mathrm{s}}: E(x)>\overline{\bar{r}}\right\} \neq \emptyset$.

Since the map $E$ is continuous, $E\left(x_{0}\right)$ is equal to $\overline{\bar{r}}$.

Let $x_{0} \in W_{\phi, c_{i}}^{\mathrm{s}}$. The definition of $K_{c_{i}, m}$ implies that $x_{0} \in K_{c_{i}, 0}$. Assume, by contradiction, that $x_{0} \in \operatorname{int}_{\text {rel }} K_{c_{i}, 0}$. Then there exists $t_{0}>0$ such that $\phi\left(x_{0},-t\right) \in K_{c_{i}, 0}$ for every $t \leq t_{0}$. However, for those $t$,

$$
E\left(\phi\left(x_{0},-t\right)\right)>E\left(\phi\left(x_{0}, 0\right)\right)=E\left(x_{0}\right)=\overline{\bar{r}} .
$$

This means that $\phi\left(x_{0}, t\right) \notin K_{c_{i}, 0}$, a contradiction.

The property of $K_{c_{i}, 1}$ can be proved in the same way.

(iii) Let $x_{0} \in \partial_{\text {rel }} K_{c_{i}, 0}$. Since the potential decreases along orbits, $E\left(\phi\left(x_{0}, t\right)\right)<E\left(x_{0}\right)$ for every $t>0$. Hence $\phi\left(x_{0}, t\right) \in K_{c_{i}, 0}$ by the definition of $K_{c_{i}, m}$.

If $x_{0} \in \operatorname{int}_{\text {rel }} K_{c_{i}, 0}$, then $E\left(x_{0}\right)<\overline{\bar{r}}$. Since the set $K_{c_{i}, 0}=B_{\text {lev }}\left(c_{i}, \overline{\bar{r}}\right)$ is homeomorphic to a ball $B_{\text {rel }}\left(c_{i}, R\right)$ such that $K_{c_{i}, 0} \subset B_{\text {rel }}\left(c_{i}, R\right)$ (see Corollary 4.4), the set $\left\{\phi\left(x_{0}, t\right): t>0\right\}$ would intersect the boundary $\partial_{\text {rel }} K_{c_{i}, 0}$ if the semiorbit $\phi\left(x_{0}, t\right), t>0$ were not included in $K_{c_{i}, 0}$. However this is impossible because the potential on the boundary of the set $K_{c_{i}, 0}$ is greater than in the interior.

The property of $K_{c_{i}, 1}$ can be shown in the same way.

(iv) Define $\tau: \overline{\mathbb{R}} \ni t \mapsto \tau(t) \in[-1,1]$ by

$$
\tau(t)= \begin{cases}-1 & \text { if } t=-\infty \\ t /(1+|t|) & \text { if } t \in \mathbb{R}, \\ 1 & \text { if } t=\infty\end{cases}
$$

Let $x_{1}, x_{2} \in K_{c_{i}, m}$, where $m$ equals 1 or 2 . Then the formulas

$$
\gamma\left(\frac{1}{2} \tau(t)\right)=\phi\left(x_{1}, t\right) \quad \text { and } \quad \gamma\left(1-\frac{1}{2} \tau(t)\right)=\phi\left(x_{2}, t\right)
$$

define an arc from $x_{1}$ to $x_{2}$; the arc is included in $K_{c_{i}, m}$ by Lemma 4.5(ii).

(v) The assertion follows from the compactness of $\mathcal{M}$. Assume, by contradiction, that $\inf \left\{\varrho_{\mathcal{M}}(x, y): x \in K_{c_{i}, 1}, y \in K_{c_{i}, 0}\right\}=0$. Then there exists a sequence $\left\{x_{n}\right\} \subset K_{c_{i}, 1}$ which converges to a point $x_{0} \in \partial_{\text {rel }} K_{c_{i}, 0}$. But $E\left(x_{0}\right)=\overline{\bar{r}}$ and $E\left(x_{n}\right) \leq \bar{r}$, which is a contradiction because $\bar{r}<\overline{\bar{r}}$ and $E$ is continuous.

The following lemma is necessary for the construction of a global homeomorphism conjugating $\phi_{h}$ and $\psi_{h}$. 
LEMMA 4.6. Let a be an attracting fixed point of the flow $\phi$ on a twodimensional manifold $\mathcal{M}$. Let, furthermore, $s_{k}, k \in\{1, \ldots, K\}$, be saddle points whose stable manifolds intersect the stable manifold of a. Then there exists a neighbourhood $V_{a}$ of a and a homeomorphism $g_{a}$ defined on this neighbourhood locally conjugating the flows $\left(\mathcal{M}, \phi_{h}\right)$ and $\left(\mathcal{M}, \psi_{h}\right)$ such that for every $k \in\{1, \ldots, K\}$,

$$
g_{a}\left(W_{\phi_{h}}^{\mathrm{u}}\left(s_{k}\right) \cap V_{a}\right) \subset W_{\psi_{h}}^{\mathrm{u}}\left(s_{k}\right) .
$$

Proof. We first record some facts and introduce a few definitions. As $\mathcal{M}$ is two-dimensional, the components of the unstable manifolds $W_{\phi_{h}}^{\mathrm{u}}\left(s_{k}\right)$ of the saddle points are curves with a common end at $a$. Choose a level set of $E$ on which $E>E(a)$ and such that its connected component is included both in $W_{\phi_{h}}^{\mathrm{s}}\left(a_{i}\right)$ and in a sufficiently small neighbourhood of $a$. Denote this component by $F_{1}$. Since near an attracting singularity a level is homeomorphic to a circle (see Corollary 4.4), this level can be parametrized as $F_{1}=F_{1}(\tau)$ with $F_{1}(0)=F_{1}(1), \tau \in[0,1]$, where the map $F_{1}$ is continuous. The set $W_{\phi}^{\mathrm{u}}\left(s_{k}\right) \backslash\left\{s_{k}, a\right\}$ has two connected components, each being an orbit of $\phi$. As already mentioned, the curve $F_{1}$ is homeomorphic to a circle and the value of the potential decreases along an orbit. Thus, every connected component of $\bigcup_{k=1}^{K}\left(W_{\phi_{h}}^{\mathrm{u}}\left(s_{k}\right) \backslash\left\{s_{k}, a\right\}\right)$ intersects $F_{1}$ in exactly one point. Let there be $M$ such components and let they be numbered from 0 to $M-1$ according to the increasing $\tau$, say $W_{\phi_{h}, m}^{\mathrm{u}}, m=0, \ldots, M-1$.

Set $F_{2}:=\phi_{h}\left(F_{1}\right)$. The curve $F_{2}$ does not intersect $F_{1}$ and is homeomorphic to $F_{1}$. The point $a$ lies in the interior of the domain bounded by $F_{2}$. Therefore the set

$$
P_{F_{1}, F_{2}}:=\left\{\phi(x, t): x \in F_{1}, t \in[0, h]\right\}
$$

is homeomorphic to a closed annulus $B(a, R) \backslash$ int $B(a, r)$, where $r<R$. Define

$$
\begin{gathered}
\delta_{m}:=W_{\phi_{h}, m}^{\mathrm{u}} \cap P_{F_{1}, F_{2}}, \quad m=0, \ldots, M-1, \\
\Lambda:[0,1] \times[0, h] \ni\{\tau, t\} \mapsto \Lambda(\tau, t)=\phi\left(F_{1}(\tau), t\right) \in P_{F_{1}, F_{2}} .
\end{gathered}
$$

The mapping $\Lambda$ is a homeomorphism because it is a superposition of homeomorphisms and there exists a finite sequence $\tau_{0}=0, \tau_{1}, \ldots, \tau_{M-1}$ such that $\Lambda\left(\tau_{m},[0, h]\right)=\delta_{m}$.

Now, we begin the proof of Lemma 4.6.

STEP 1: Construction on fragments of $W_{\phi_{h}}^{\mathrm{u}}\left(s_{k}\right)$. Let $g_{s_{k}}$ denote a homeomorphism locally conjugating $\phi_{h}$ and $\psi_{h}$ near $s_{k}$ and let $\phi_{-h}$ be the inverse of $\phi_{h}$. The formula

$$
g_{s_{k}, a}(x):=\left(\psi_{h}^{n_{0}(x)} \circ g_{s_{k}} \circ \phi_{-h}^{n_{0}(x)}\right)(x)
$$

defines a homeomorphism conjugating $\phi_{h}$ and $\psi_{h}$ on $\delta_{m}$. The natural number 
$n_{0}(x)$ is chosen in such a way that $\phi_{-h}^{n_{0}(x)}(x) \in K_{s_{k}, 1}$ and $\phi_{-h}^{n_{0}(x)-1}(x) \in$ $\operatorname{int}_{\text {rel }}\left(K_{s_{k}, 0} \backslash K_{s_{k}, 1}\right)$. Note that the parameter $m$ uniquely identifies the saddle point $s_{k}$.

The mapping

$$
g_{a}:=g_{s_{k}, a}, \quad x \in \delta_{m},
$$

defines a homeomorphism conjugating $\phi_{h}$ and $\psi_{h}$ on $\bigcup_{m=0}^{M-1} \delta_{m}$. The mappings $\psi_{h}, g_{s_{k}}$ and $g_{a}$ are continuous in $h$ and each is the identity for $h=0$.

STEP 2: Construction on a fragment of the annulus. Set

$\gamma_{1, m}:=\left\{x=F_{1}(\tau): \tau \in\left[\tau_{m}, \tau_{m+1}\right]\right\}, \gamma_{2, m}:=\left\{x=F_{2}(\tau): \tau \in\left[\tau_{m}, \tau_{m+1}\right]\right\}$, where $\tau_{m}$ is the value of $\tau$ for which

$$
F_{j}\left(\tau_{m}\right)=F_{j} \cap W_{\phi_{h}, m}^{\mathrm{u}}=: x_{j}(m), \quad j \in\{1,2\} .
$$

Note that $\gamma_{j, m}, j=1,2, m=0, \ldots, M-1$, is a curve with end points $x_{j}(m), x_{j}(m+1)$ whereas $\delta_{m}$ is a curve with end points $x_{1}(m), x_{2}(m)$. All these curves are homeomorphic to line segments. This implies that $\gamma_{1, m} \cup$ $\gamma_{2, m} \cup \delta_{m} \cup \delta_{m+1}$ bounds a simply connected domain in $\mathbb{R}^{2}$. By the Riemann Theorem this domain is homeomorphic to a ball and hence to a rectangle. By Lemma 4.1 there exists a homeomorphism

$$
\Lambda_{m}:=\left.\Lambda\right|_{\left[\tau_{m}, \tau_{m+1}\right] \times[0, h]}
$$

from $\left[\tau_{m}, \tau_{m+1}\right] \times[0, h]$ onto the closed domain $\mathrm{Sq}_{\gamma_{1, m}, \gamma_{2, m}, \delta_{m}, \delta_{m+1}}$ bounded by the curves $\gamma_{1, m}, \gamma_{2, m}, \delta_{m}$ and $\delta_{m+1}$. The mapping $\Lambda$ has the following properties:

$$
\begin{aligned}
\Lambda_{m}\left(\tau_{m},[0, h]\right) & =\delta_{m}, \\
\Lambda_{m}\left(\left[\tau_{m}, \tau_{m+1}\right], 0\right) & =\gamma_{1, m}, \\
\Lambda_{m}\left(\left[\tau_{m}, \tau_{m+1}\right], h\right) & =\gamma_{2, m} .
\end{aligned}
$$

Perform a similar construction for the cascade $\psi_{h}$. Set

$$
x_{j}^{*}(m):=g_{a}\left(x_{j}(m)\right) .
$$

Let a curve $F_{1}^{*}:=g_{a}\left(F_{1}\right)$ parametrized by $\tau$ be homeomorphic to a circle and such that $a$ is in the interior of the simply connected domain bounded by $F_{1}^{*}$. Let, furthermore, the points $x_{1}^{*}(m), m \in\{0, \ldots, M-1\}$, lie in $F_{1}^{*}$ and let the parametrization have the property

$$
F_{1}^{*}\left(\tau_{m}\right)=x_{1}^{*}(m), \quad m=0, \ldots, M-1 .
$$

Let $F_{2}^{*}:=\psi_{h}\left(F_{1}^{*}\right)$ and suppose $\psi_{h}\left(F_{1}^{*}(\tau)\right)=F_{2}^{*}(\tau)$ for all $\tau$. Set

$$
\delta_{m}^{*}:=g_{a}\left(\delta_{m}\right),
$$

and let $\delta_{m}^{*}$ be parametrized in such a way that

$$
\delta_{m}^{*}\left(h_{0}\right):=g_{a}\left(\delta_{m}\left(h_{0}\right)\right), \quad h_{0} \in[0, h] .
$$


The homeomorphism $g_{a}$ is a continuous function of $h$ and is the identity for $h=0$, so for sufficiently small $h$, the curve $\delta_{m}^{*}$ lies near $\delta_{m}$ and does not intersect any other $\delta_{m^{\prime}}, m^{\prime} \neq m$. Thus, if $m$ increases then so does the value of $\tau$ which parametrizes $F_{1}^{*}$. Therefore, $F_{1}^{*}$ can be reparametrized in such a way that the values of $\tau$ at $x_{j}(m)$ equal those at $x_{j}^{*}(m), m=0, \ldots, M-1$. Define

$$
\begin{aligned}
& \gamma_{1, m}^{*}:=\left\{x \in F_{1}^{*}(\tau): \tau \in\left[\tau_{m}, \tau_{m+1}\right]\right\}, \\
& \gamma_{2, m}^{*}:=\left\{x \in F_{2}^{*}(\tau): \tau \in\left[\tau_{m}, \tau_{m+1}\right]\right\} .
\end{aligned}
$$

Arguing as for $\phi_{h}$, we can show that the domain bounded by $F_{1}^{*}$ and $F_{2}^{*}$ is homeomorphic to an annulus whereas the domain $\mathrm{Sq}_{\gamma_{1, m}^{*}, \gamma_{2, m}^{*}, \delta_{m}^{*}, \delta_{m+1}^{*}}$ bounded by $\gamma_{1, m}^{*}, \gamma_{2, m}^{*}, \delta_{m}^{*}$ and $\delta_{m+1}^{*}$ is homeomorphic to a rectangle. By Lemma 4.1 the homeomorphism can be chosen to map the vertices of the rectangle to the points $x_{1}^{*}(m), x_{2}^{*}(m), x_{1}^{*}(m+1)$ and $x_{2}^{*}(m+1)$. Denote it by

$$
\xi_{m}: \mathrm{Sq}_{\gamma_{1, m}^{*}, \gamma_{2, m}^{*}, \delta_{m}^{*}, \delta_{m+1}^{*}} \rightarrow\left[\tau_{m}, \tau_{m+1}\right] \times[0, h] .
$$

It has the following properties:

$$
\begin{aligned}
\xi_{m}\left(\gamma_{1, m}^{*}\right) & =\left[\tau_{m}, \tau_{m+1}\right] \times\{0\}, \\
\xi_{m}\left(\gamma_{2, m}^{*}\right) & =\left[\tau_{m}, \tau_{m+1}\right] \times\{h\}, \\
\xi_{m}\left(\delta_{m}^{*}\right) & =\left\{\tau_{m}\right\} \times[0, h] .
\end{aligned}
$$

Furthermore, $\xi_{m}$ can be constructed in such a way that on the curves $\delta_{m}^{*}$ it is consistent with the mapping $g_{m, a}$ (the parameter $m$ determines the saddle point $s_{k}$ uniquely):

$$
g_{m, a}\left(\phi\left(x_{m}, t\right)\right)=\xi_{m}\left(\tau_{m}, t\right), \quad t \in[0, h],
$$

$$
g_{m+1, a}\left(\phi\left(x_{m}+1, t\right)\right)=\xi_{m}\left(\tau_{m+1}, t\right), \quad t \in[0, h] .
$$

This can be shown in the following way. Equations (13) and (14) imply that on the vertical sides $\tau_{m} \times[0, h]$ and $\tau_{m+1} \times[0, h]$ the parametrization is settled by increasing homeomorphisms $f_{1}:[0, h] \rightarrow[0, h]$ and $f_{2}:[0, h] \rightarrow[0, h]$. We will show that the mapping

$$
\begin{aligned}
g:\left[\tau_{m}, \tau_{m+1}\right] \times[0, h] \ni\{\tau, t\} & \mapsto \\
g(\tau, t) & =\left(g_{1}(\tau, t), g_{2}(\tau, t)\right) \in\left[\tau_{m}, \tau_{m+1}\right] \times[0, h]
\end{aligned}
$$

defined by

$$
g_{1}(\tau, t)=\tau, \quad g_{2}(\tau, t)=\frac{\tau_{m+1}-\tau}{\tau_{m+1}-\tau_{m}} \cdot f_{1}(t)+\frac{\tau-\tau_{m}}{\tau_{m+1}-\tau_{m}} \cdot f_{2}(t)
$$

is a homeomorphism with the required properties. We will prove that it transforms the rectangle $\left[\tau_{m}, \tau_{m+1}\right] \times[0, h]$ onto itself. Fix $\tau_{0} \in\left[\tau_{m}, \tau_{m+1}\right]$ 
and $t_{0} \in[0, h]$. Without losing generality we can assume that $f_{1}\left(t_{0}\right) \leq f_{2}\left(t_{0}\right)$. Then

$$
g_{2}\left(\tau_{0}, t_{0}\right) \geq \frac{\tau_{m+1}-\tau_{0}}{\tau_{m+1}-\tau_{m}} \cdot f_{1}\left(t_{0}\right)+\frac{\tau_{0}-\tau_{m}}{\tau_{m+1}-\tau_{m}} \cdot f_{1}\left(t_{0}\right)=f_{1}\left(t_{0}\right) \in[0, h]
$$

and

$$
g_{2}\left(\tau_{0}, t_{0}\right) \leq \frac{\tau_{m+1}-\tau_{0}}{\tau_{m+1}-\tau_{m}} \cdot f_{2}\left(t_{0}\right)+\frac{\tau_{0}-\tau_{m}}{\tau_{m+1}-\tau_{m}} \cdot f_{2}\left(t_{0}\right)=f_{2}\left(t_{0}\right) \in[0, h] .
$$

Furthermore, $g_{2}\left(\tau_{m}, \cdot\right)=f_{1}(\cdot)$ and $g_{2}\left(\tau_{m+1}, \cdot\right)=f_{2}(\cdot)$. The jacobian

$$
\operatorname{jac} g=\left(\begin{array}{ll}
\partial g_{1} / \partial \tau & \partial g_{1} / \partial t \\
\partial g_{2} / \partial \tau & \partial g_{2} / \partial t
\end{array}\right)=\frac{\tau_{m+1}-\tau}{\tau_{m+1}-\tau_{m}} \cdot \frac{d f_{1}(t)}{d t}+\frac{\tau-\tau_{m}}{\tau_{m+1}-\tau_{m}} \cdot \frac{d f_{2}(t)}{d t}
$$

is positive at each point because both components are nonnegative $\left(f_{1}\right.$ and $f_{2}$ are increasing) and do not equal zero simultaneously.

The superposition

$$
\Upsilon_{m}=\Lambda_{m} \circ g \circ \xi_{m}
$$

is a homeomorphism transforming the closure of $\mathrm{Sq}_{\gamma_{1, m}^{*}, \gamma_{2, m}^{*}, \delta_{m}^{*}, \delta_{m+1}^{*}}$ onto the closure of $\mathrm{Sq}_{\gamma_{1, m}, \gamma_{2, m}, \delta_{m}, \delta_{m+1}}$ in such a way that for each $x \in \delta_{m} \cup \delta_{m+1}$,

$$
\Upsilon_{m}(x)=g_{m, a}(x) .
$$

STEP 3: Construction on the annulus. By (13) and (14), $\Upsilon_{m}(x)=\Upsilon_{m+1}(x)$ for each $x \in \delta_{m+1}$ and $\Upsilon_{0}(x)=\Upsilon_{M-1}(x)$ for $x \in \delta_{0}$. Therefore the mapping defined as

$$
\Upsilon(x)=\Upsilon_{m}(x) \quad \text { for } x \in \mathrm{Sq}_{\gamma_{1, m}^{*}, \gamma_{2, m}^{*}, \delta_{m}^{*}, \delta_{m+1}^{*}} \text { and } m \in\{0,1, \ldots, M-1\}
$$

is a homeomorphism transforming the "annulus" $P_{F_{1}^{*}, F_{2}^{*}}$ onto the "annulus" $P_{F_{1}, F_{2}}$.

STEP 4: Construction on the neighbourhood of a. Extend $\Upsilon$ to the whole neighbourhood of $a$. Let $y \in P_{F_{1}^{*}, F_{2}^{*}}$, and define $x \in V_{a}$ by

$$
x=\psi_{h}^{k(x)}(y), \quad k \in \mathbb{N} .
$$

Set

$$
\widetilde{\Upsilon}_{a}(x):=\left\{\begin{aligned}
&\left(\psi_{h}^{-k(x)} \circ \Upsilon \circ \phi_{h}^{k(x)}\right)(x) \\
& \text { if } \exists y \in P_{F_{1}^{*}, F_{2}^{*}}: x=\psi_{h}^{k(x)}(y), k \in \mathbb{N}, \\
& a \text { for } x=a .
\end{aligned}\right.
$$

For points of the curve $F_{2}^{*}$ the mapping $\widetilde{\Upsilon}_{a}$ is defined in two ways. First, $F_{2}^{*} \subset P_{F_{1}^{*}, F_{2}^{*}}$ so we can take zero as the value of $k$. On the other hand we can take $k=1$ because every point of $F_{2}^{*}$ is the image of a point of $F_{1}^{*}$. However, both $\phi_{h}$ and $\psi_{h}$ preserve values of $\tau$ so for each $x \in F_{2}^{*}$ both the ways give the same image. This also implies that $\widetilde{\Upsilon}_{a}$ is continuous on $F_{2}^{*}$. 
To prove the continuity of $\widetilde{\Upsilon}_{a}$ at $a$ consider a sequence $\left\{x_{n}\right\}$ converging to $a$. For each $n$ there exists a natural number $k_{n}$ such that $\psi_{h}^{-k_{n}}\left(x_{n}\right) \in P_{F_{1}^{*}, F_{2}^{*}}$. Furthermore, $k_{n} \rightarrow \infty$. Thus $\widetilde{\Upsilon}_{a}\left(x_{n}\right) \rightarrow a$. Hence, the function

$$
\widetilde{\Upsilon}_{a}: P_{F_{1}^{*}, F_{2}^{*}} \rightarrow P_{F_{1}, F_{2}}
$$

is a homeomorphism.

Since on the sets $\delta_{m}, m=0, \ldots, M-1$ (see (11)) the mapping $\Upsilon$ is defined by the local conjugating homeomorphism $g_{s_{k}}$ (see (12) and (15)) which transforms $W_{\phi_{h}}^{\mathrm{u}}\left(s_{k}\right)$ onto $W_{\psi_{h}}^{\mathrm{u}}\left(s_{k}\right)$, the inclusion (10) is satisfied.

Introduce the following notations:

$\mathcal{D}_{\phi_{h}}$ - the set of all repelling points,

$\mathcal{S}_{\phi_{h}}$ - the set of all saddle points and the attracting points which are not contained in the closure of the unstable manifold of any saddle point,

$\mathcal{P}_{\phi_{h}}$ - the set of attracting points which are contained in the closure of the unstable manifold of a saddle point.

Define

$$
W_{\phi_{h}}^{\mathrm{s}}\left(\mathcal{S}_{\phi_{h}}\right):=\bigcup_{c \in \mathcal{S}_{\phi_{h}}} W_{\phi_{h}}^{\mathrm{s}}(c), \quad W_{\phi_{h}}^{\mathrm{s}}\left(\mathcal{P}_{\phi_{h}}\right):=\bigcup_{c \in \mathcal{P}_{\phi_{h}}} W_{\phi_{h}}^{\mathrm{s}}(c),
$$

and $\Theta: W_{\phi_{h}}^{\mathrm{s}}\left(\mathcal{S}_{\phi_{h}}\right) \rightarrow \mathcal{M}$ by

$$
\Theta(x)= \begin{cases}\left(\psi_{-h}^{n_{0}} \circ g_{c} \circ \phi_{h}^{n_{0}}\right)(x) & \text { for } x \in W_{\phi_{h}}^{\mathrm{s}}(c) \backslash\{c\}, \\ x & \text { for } x=c,\end{cases}
$$

where $c \in \mathcal{S}_{\phi_{h}}, g_{c}$ is a local homeomorphism conjugating the flows $\phi_{h}$ and $\psi_{h}$ on a neighbourhood of $c$ and $\psi_{-h}$ is the inverse of $\psi_{h}$. If $x$ is in one of the sets $K_{c, 1}$, then the natural number $n_{0}=n_{0}(x)$ is zero. In the other case it is chosen in such a way that $\phi_{h}^{n_{0}}(x) \in K_{c, 1}$ and $\phi_{h}^{n_{0}-1}(x) \in \operatorname{int}_{\mathrm{rel}}\left(K_{c, 0} \backslash K_{c, 1}\right)$.

Define $\alpha: \mathcal{M} \rightarrow \mathcal{M}$ as follows:

$$
\alpha(x)= \begin{cases}\Theta(x) & \text { for } x \in W_{\phi_{h}}^{\mathrm{s}}\left(\mathcal{S}_{\phi_{h}}\right), \\ \widetilde{\Upsilon}_{a}(x) & \text { for } x \in W_{\phi_{h}}^{\mathrm{s}}\left(\mathcal{P}_{\phi_{h}}\right), a \in \mathcal{P}_{\phi_{h}}, \\ x & \text { for } x \in \mathcal{D}_{\phi_{h}} .\end{cases}
$$

Every nonrepelling point on a compact manifold is contained in a stable manifold of an attracting or saddle singularity. Thus (18) defines $\alpha$ on the whole $\mathcal{M}$. In the next section it is shown that $\alpha$ is a homeomorphism globally conjugating the flows $\left(\mathcal{M}, \phi_{h}\right)$ and $\left(\mathcal{M}, \psi_{h}\right)$.

LEMMA 4.7. For all positive constants $\varepsilon$, there exists a positive constant $h_{0}$ such that for all $x \in \mathcal{M}, t \in \mathbb{R}$ and $0<h<h_{0}$,

$$
\varrho_{\mathcal{M}}(\phi(x, t), \phi(x, t+h))<\varepsilon .
$$

The proof of this simple lemma is omitted (it can be found in [Bie]). 
LEMMA 4.8. Let a be an attracting fixed point of the flow $\phi$, and $p_{1}, p_{2}$ repelling fixed points, $p_{1} \neq p_{2}$. Assume that $p_{1}, p_{2} \in \mathrm{cl} W_{\phi}^{\mathrm{s}}(a)$. Then there exist saddle points $q_{1}, q_{2}$, not necessarily different, such that $p_{1} \in \mathrm{cl} W_{\phi}^{\mathrm{s}}\left(q_{1}\right)$ and $p_{2} \in \mathrm{cl} W_{\phi}^{\mathrm{s}}\left(q_{2}\right)$.

Proof. Since $p_{1}, p_{2} \in \partial W_{\phi}^{\mathrm{s}}(a)$, there exist $y_{1}$ and $y_{2}$ such that

$$
\begin{aligned}
& \lim _{t \rightarrow \infty} \phi\left(y_{1}, t\right)=a, \quad \lim _{t \rightarrow-\infty} \phi\left(y_{1}, t\right)=p_{1}, \\
& \lim _{t \rightarrow \infty} \phi\left(y_{2}, t\right)=a, \quad \lim _{t \rightarrow-\infty} \phi\left(y_{2}, t\right)=p_{2} .
\end{aligned}
$$

Let $z_{1} \in \operatorname{orb}\left(y_{1}\right) \cap V_{a}$ and $z_{2} \in \operatorname{orb}\left(y_{2}\right) \cap V_{a}$, where orb $(y)$ denotes the orbit of $y$, and let $V_{a} \subset W_{\phi, a}^{\mathrm{s}}$ be a neighbourhood of $a$. The manifold $\mathcal{M}$ is locally arcwise connected, so let

$$
l:[0,1] \ni \tau \mapsto l(\tau) \in X
$$

be a closed arc included in $V_{a}$, avoiding $a$ and such that $l(0)=z_{1}, l(1)=z_{2}$. Since $z_{1}$ and $z_{2}$ lie in the unstable manifolds of $p_{1}$ and $p_{2}$ respectively, the boundaries of the unstable manifolds intersect $l$ in points $w_{1}$ and $w_{2}$, not necessarily different. As the unstable manifolds of repelling points are open and $\mathcal{M}$ is compact, the points $w_{1}, w_{2}$ are contained in the unstable manifolds of saddle points $q_{1}, q_{2}$, not necessarily different. From the $\lambda$-lemma it follows that for every neighbourhood $V_{q_{i}}$ of $q_{i}$ there exists $t>0$ such that

$$
\phi\left(l \cap W_{\phi}^{\mathrm{u}}\left(p_{1}\right),-t\right) \cap V_{q_{1}} \neq 0 .
$$

We have

$$
\left(W_{\phi}^{\mathrm{s}}\left(q_{1}\right) \backslash\left\{q_{1}\right\}\right) \cap V_{q_{1}} \neq 0 .
$$

Thus, for every $\varepsilon>0$ there exists $t>0$ such that $\phi\left(l \cap W_{\phi}^{\mathrm{u}}\left(p_{1}\right),-t\right)$ intersects the $\varepsilon$-envelope of $W_{\phi}^{\mathrm{s}}\left(q_{1}\right)$. This implies that there exists $u \in W_{\phi}^{\mathrm{s}}\left(q_{1}\right)$ such that

$$
\lim _{t \rightarrow-\infty} \phi(u, t)=p_{1} .
$$

Therefore, the point $p_{1} \in \operatorname{cl} W_{\phi}^{\mathrm{u}}\left(q_{1}\right)$. The same can be said about the points $q_{2}$ and $p_{2}$.

Corollary 4.9. Let $p$ be a repelling point which is not contained in the closure of the stable manifold of any saddle point. Then there exists only one attracting point a such that $p \in \operatorname{cl} W_{\phi}^{\mathrm{s}}(a)$.

This follows easily from Lemma 4.8 .

Corollary 4.10. Let $\mathcal{M}=\mathcal{S}^{n}, n>1$. Then, for every $h>0$, the cascade $\left(\mathcal{S}^{n}, \phi_{h}\right)$ has no saddle-saddle connections and, for sufficiently small $h$, the cascade $\left(\mathcal{S}^{n}, \psi_{h}\right)$ has no such connections either.

Proof. By the assumptions of Theorem 2.1, the flow $\left(\mathcal{S}^{n}, \phi\right)$ has no saddle-saddle connections. Thus each saddle point $q_{i}, i=1, \ldots, I$, has a 
neighbourhood $U_{q_{i}}$ such that every point $x_{0} \in U_{q_{i}}$ lies in $W_{\phi}^{\text {s }}\left(q_{i}\right)$ or in $W_{\phi}^{\mathrm{s}}\left(a_{j}\right)$ for an attracting point $a_{j}$. For every $x \in \mathcal{S}^{n}$ and $h>0$,

$$
\lim _{t \rightarrow \infty} \phi(x, t)=\lim _{n \rightarrow \infty} \phi_{h}^{n}(x),
$$

hence $x_{0} \in W_{\phi_{h}}^{\mathrm{s}}\left(q_{i}\right)$ or $x_{0} \in W_{\phi_{h}}^{\mathrm{s}}\left(a_{j}\right)$.

Define

$$
P_{i}:=\left\{x \in U_{q_{i}}: \forall_{n \in\{1,2, \ldots\}} \psi_{h}^{n}(x) \notin U_{q_{i}}\right\}
$$

and let $U_{q_{i}} \subset \operatorname{int} V_{q_{i}}$, where $V_{q_{i}}$ is a neighbourhood on which the cascades $\phi_{h}$ and $\psi_{h}$ are locally conjugate. Choose real numbers $R$ and $h_{i}$ such that $B\left(q_{i}, R\right) \subset \operatorname{int} U_{q_{i}}$ and $P_{i} \cap B\left(q_{i}, R / 2\right) \neq \emptyset$ for every $h \in\left[0, h_{i}\right]$.

Step 1: We will show that $P_{i} \cap W_{\phi}^{\mathrm{s}}\left(q_{i}\right)=\emptyset$ for sufficiently small $h_{i}$. Define

$$
t_{i}:=\sup _{x \in U_{q_{i}} \cap W_{\phi}^{s}\left(q_{i}\right)}\left\{t: \phi(x, t) \in B\left(q_{i}, R / 4\right) \backslash B\left(q_{i}, R / 8\right)\right\} .
$$

It is obvious that $t_{i} \in(0, \infty)$. Suppose $h_{i}$ is such that there exists a natural number $n_{1}$ with $t_{i}=n_{1} h_{i}$ and

$$
\xi h_{i}<R / 4
$$

where $\xi$ is the constant from (3). Since there are only a finite number of singularities, we can take $\xi$ as the maximum value of the constants of all saddle points.

Let $x \in U_{q_{i}} \cap W_{\phi}^{\mathrm{s}}\left(q_{i}\right)$. Then

$$
\phi_{h_{i}}^{n_{1}}(x) \in B\left(q_{i}, R / 4\right)
$$

and by (3) and the choice of $h_{i}$,

$$
\varrho_{\mathcal{M}}\left(\phi_{h_{i}}^{n_{1}}(x), \psi_{h_{i}}^{n_{1}}(x)\right)<R / 4,
$$

or equivalently

$$
\psi_{h_{i}}^{n_{1}}(x) \in B\left(q_{i}, R / 2\right) .
$$

Since $P_{i} \cap B\left(q_{i}, R / 2\right)=\emptyset$, the point $x$ is not in $P_{i}$.

SteP 2: Proof of lemma. Let $x \in P_{i}$. The first step implies that $x \notin W_{\phi}^{\mathrm{s}}\left(q_{i}\right)$. Since there are no saddle-saddle connections, the point $x$ is in $W_{\phi}^{\mathrm{s}}\left(a_{j}\right)$ for an attracting point $a_{j}$. Let $V_{a_{j}}$ be the neighbourhood of $a_{j}$ on which $\phi_{h}$ and $\psi_{h}$ are topologically conjugate. Decompose the set $P_{i}$ into disjoint components in the following way:

$$
P_{i j}:=\left\{x \in P_{i}: x \in W_{\phi}^{\mathrm{s}}\left(a_{j}\right)\right\} .
$$

Let, furthermore,

$$
t_{i j}:=\sup \left\{t: \phi(x, t) \in B\left(a_{j}, r / 2\right) \backslash B\left(a_{j}, r / 4\right), x \in P_{i j}\right\},
$$

where $r$ is chosen in such a way that $B\left(a_{j}, r\right) \subset V_{a_{j}}$. It is obvious that $t_{i j} \in(0, \infty)$. Denote by $\bar{e}_{n}$ the error after the $n$th step of the Euler method. 
Choose $h_{i j}$ so small that $\phi_{h}$ and $\psi_{h}$ are topologically conjugate on $V_{a_{j}}$ and $\xi h_{i j}<r / 2$. Moreover, let $t_{i j}=n_{2} h_{i j}, n_{2} \in\{1,2, \ldots\}$. Then $\phi\left(x, t_{i j}\right) \in$ $B\left(a_{j}, r / 2\right)$ for every $x \in P_{i j}$. By (3) and the choice of $h_{i j}$,

$$
\bar{e}_{n}:=\varrho_{\mathcal{M}}\left(\phi_{h_{i j}}^{n_{2}}(x), \psi_{h_{i j}}^{n_{2}}(x)\right)<r / 2 .
$$

Hence

$$
\psi_{h_{i j}}^{n_{2}}(x) \in B\left(a_{j}, r\right) .
$$

Assuming that there are $I$ saddle points and $J$ attracting points, set

$$
h_{0}:=\min _{i \in\{1, \ldots, I\}, j \in\{1, \ldots, J\}}\left\{h_{i j}\right\} .
$$

As $V_{a_{j}} \subset W_{\phi_{h_{0}}}^{\mathrm{s}}\left(a_{j}\right)$ and the cascades are locally topologically conjugate, we have $V_{a_{j}} \subset W_{\psi_{h}}^{\mathrm{s}}\left(a_{j}\right)$ for every $h \in\left[0, h_{0}\right]$. Hence

$$
\psi_{h}^{n_{2}}(x) \in W_{\psi_{h}}^{\mathrm{s}}\left(a_{j}\right) .
$$

Therefore, every $x \in U_{q_{i}}$ is either in $W_{\psi_{h}}^{\mathrm{s}}\left(q_{i}\right)$ or in $W_{\psi_{h}}^{\mathrm{s}}\left(a_{j}\right)$, where $a_{j}$ is an attracting point. This implies that $\psi_{h}$ has no saddle-saddle connections.

Remark. By Lemma 4.10 and Theorem 4.2 , if $\left(\mathcal{S}^{n}, \phi\right)$ is a gradient Morse-Smale system, then so are the cascade $\left(\mathcal{S}^{n}, \phi_{h}\right)$ (for every positive $h$ ) and $\left(\mathcal{S}^{n}, \psi_{h}\right)$ (for sufficiently small $h$ ).

COROLlary 4.11. Let $U_{q_{i}}$ be a neighbourhood of saddle point $q_{i}$ on which the cascades $\phi_{h}$ and $\psi_{h}$ are conjugate by a homeomorphism $g_{q_{i}}: U_{q_{i}} \rightarrow$ $g_{q_{i}}\left(U_{q_{i}}\right)$. Then there exists a constant $h_{0}>0$ such that for every $h \in\left(0, h_{0}\right)$ and $x \in U_{q_{i}} \cap W_{\phi_{h}}^{\mathrm{u}}\left(q_{i}\right)$ if $x \in W_{\phi_{h}}^{\mathrm{s}}\left(a_{j}\right)$, where $a_{j}$ is an attracting fixed point, then $g_{i}(x) \in W_{\psi_{h}}^{\mathrm{s}}\left(a_{j}\right)$.

Proof. Step 1. Define

$$
H_{i j}:=W_{\phi_{h}}^{\mathrm{u}}\left(q_{i}\right) \cap\left(B\left(q_{i}, r_{i}\right) \backslash \operatorname{int} B\left(q_{i}, r_{i} / 2\right)\right) \cap W_{\phi_{h}}^{\mathrm{s}}\left(a_{j}\right) .
$$

First, it will be shown that there exists $h_{0}>0$ so small that for every $h \in\left(0, h_{0}\right)$ and every $x \in H_{i j}$ we have $g_{i}(x) \in W_{\psi_{h}}^{\mathrm{s}}\left(a_{j}\right)$. The radius $r_{i}$ is chosen such that $B\left(q_{i}, r_{i}\right) \subset U_{q_{i}}$.

The definition (20) implies that $H_{i j}$ is closed in $\mathcal{M}$, hence compact. The stable manifold $W_{\phi_{h}}^{\mathrm{s}}\left(a_{j}\right)$ is open and $H_{i j} \subset W_{\phi_{h}}^{\mathrm{s}}\left(a_{j}\right)$. Thus, for every $x \in H_{i j}$ there exists $r_{x}>0$ such that $B\left(x, r_{x}\right) \subset W_{\phi_{h}}^{\mathrm{s}}\left(a_{j}\right)$. The set

$$
\mathcal{K}:=\left\{B\left(x, r_{x}\right) \cap W_{\phi_{h}}^{\mathrm{u}}\left(q_{i}\right)\right\}
$$

is a covering of $H_{i j}$. Thus, we can choose a finite subcovering $\mathcal{K}^{*}$. Let $r_{i j}$ be the smallest radius of the balls $B\left(x, r_{x}\right)$ used to construct $\mathcal{K}^{*}$. Take $h_{0 i j}>0$ such that $g_{q_{i}}(x) \in B\left(x, r_{i j}\right)$ for every point $x \in H_{i j}$. Then $g_{q_{i}}(x) \in W_{\phi_{h}}^{\mathrm{s}}\left(a_{j}\right)$. It can be shown (in the same way as in the second step of the proof of Corollary 4.10) that there exists $h_{1 i j}$ such that $g\left(H_{i j}\right) \subset W_{\psi_{h}}^{\mathrm{s}}\left(a_{j}\right)$ for every $h \in\left(0, h_{1 i j}\right)$. Set $h_{i j}:=\min \left\{h_{0 i j}, h_{1 i j}\right\}$. 
STEP 2. Suppose, by contradiction, that there exists $y \in W_{\phi_{h}}^{\mathrm{u}}\left(q_{i}\right) \cap$ $\operatorname{int} B\left(q_{i}, r_{i} / 2\right)$ such that $y_{1} \in W_{\phi_{h}}^{\mathrm{s}}\left(a_{j}\right)$ and $z_{1}:=g_{i}\left(y_{1}\right) \in W_{\phi_{h}}^{\mathrm{s}}\left(a_{k}\right)$, where $a_{k}$ is an attracting fixed point and $k \neq j$. Corollary 4.7 implies that, for sufficiently small $h$, there exists a natural $m_{0}$ such that

$$
\phi_{h}^{m_{0}}\left(y_{1}\right) \in B\left(q_{i}, r_{i}\right) \backslash \operatorname{int} B\left(q_{i}, r_{i} / 2\right) .
$$

As $y_{1} \in W_{\phi_{h}}^{\mathrm{s}}\left(a_{j}\right)$, the point $y_{2}:=\phi_{h}^{m_{0}}\left(y_{1}\right)$ is in $W_{\phi_{h}}^{\mathrm{s}}\left(a_{j}\right)$. Therefore, by Step $1, z_{2}:=g_{i}\left(y_{2}\right) \in W_{\psi_{h}}^{\mathrm{s}}\left(a_{j}\right)$ as $y_{2} \in P_{i j}$. However

$$
\begin{aligned}
z_{2} & :=g\left(y_{2}\right)=g\left(\phi_{h}^{m_{0}}\left(y_{1}\right)\right)=\left(g \circ \phi_{h}\right)\left(\phi_{h}^{m_{0}-1}\left(y_{1}\right)\right) \\
& =\left(\psi_{h} \circ g \circ \phi_{h}\right)\left(\phi_{h}^{m_{0}-2}\left(y_{1}\right)\right) \\
& =\left(\psi_{h} \circ \psi_{h} \circ g\right)\left(\phi_{h}^{m_{0}-2}\left(y_{1}\right)\right)=\ldots=\psi_{h}^{m_{0}}\left(g\left(y_{1}\right)\right)=\psi_{h}^{m_{0}}\left(z_{1}\right),
\end{aligned}
$$

and $\psi_{h}^{m_{0}}\left(z_{1}\right) \in W_{\psi_{h}}^{\mathrm{s}}\left(a_{k}\right)$ as $z_{1} \in W_{\psi_{h}}^{\mathrm{s}}\left(a_{k}\right)$, which leads to a contradiction.

5. Proof of Theorem. Firstly, we prove that the map $\alpha$ defined in (18) is a bijection. Then we prove that it is continuous on $\mathcal{M}$. This implies that $\alpha$ is a homeomorphism. The map $\alpha$ conjugates the cascades $\phi_{h}$ and $\psi_{h}$, which follows directly from its definition (see (16)-(18)).

Injectivity. Let $x_{1}, x_{2} \in \mathcal{S}^{2}$. If at least one of them is a repelling singularity of the system $\phi$, then $\alpha\left(x_{1}\right) \neq \alpha\left(x_{2}\right)$ by the definition of $\alpha$. Otherwise the following two cases have to be considered:

CASE 1: The points lie in different orbits. Then

$$
y_{1}:=\phi_{h}^{n_{0}\left(x_{1}\right)}\left(x_{1}\right) \neq \phi_{h}^{n_{0}\left(x_{2}\right)}\left(x_{2}\right)=: y_{2} .
$$

The system $\phi$ has only a finite number of singularities and every singularity $c_{i}$ is a fixed point of $g_{i}$. The sphere $\mathcal{S}^{2}$ is compact so every point is contained in the stable manifold of a stable point: $x_{1} \in W_{\phi}^{\mathrm{s}}\left(c_{i}\right), x_{2} \in W_{\phi}^{\mathrm{s}}\left(c_{j}\right)$.

If $i=j$, then $z_{1}:=g_{i}\left(y_{1}\right) \neq g_{i}\left(y_{2}\right)=z_{2}$ as $g_{i}$ is a bijection. Since $g_{i}$ is a local conjugating homeomorphism, the images of different orbits do not intersect. Thus $\psi_{-h}^{n_{0}\left(x_{1}\right)}\left(z_{1}\right) \neq \psi_{-h}^{n_{0}\left(x_{2}\right)}\left(z_{2}\right)$, which implies that $\alpha\left(x_{1}\right) \neq \alpha\left(x_{2}\right)$.

If $i \neq j$, then we can choose the domains $V_{i}$ of the homeomorphisms $g_{i}$ in such a way that $V_{i} \cap V_{j}=\emptyset$ and $g_{i}\left(V_{i}\right) \cap g_{j}\left(V_{j}\right)=\emptyset$ for $i \neq j$. Thus $z_{1}:=g_{i}\left(y_{1}\right) \neq g_{j}\left(y_{2}\right)=: z_{2}$. As $x_{1} \in W_{\phi_{h}, a_{i}}^{\mathrm{s}}$ and $x_{2} \in W_{\phi_{h}, a_{j}}^{\mathrm{s}}$, the points $z_{1}$ and $z_{2}$ lie in the disjoint manifolds $W_{\psi_{h}, a_{i}}^{\mathrm{s}}$ and $W_{\psi_{h}, a_{j}}^{\mathrm{s}}$ respectively. This implies that $\alpha\left(x_{1}\right) \neq \alpha\left(x_{2}\right)$.

CAsE 2: The points lie in the same orbit. We can assume that $E\left(x_{2}\right)>$ $E\left(x_{1}\right)$. Then $x_{1}=\phi_{h}^{m}\left(x_{2}\right)$ for some positive natural number $m=m\left(x_{1}, x_{2}\right)$. 
Thus

$$
\begin{aligned}
\alpha\left(x_{2}\right) & =\left(\psi_{-h}^{n_{0}\left(x_{1}\right)+m} \circ g_{c_{i}} \circ \phi_{h}^{n_{0}\left(x_{1}\right)+m}\right)\left(x_{2}\right) \\
& =\left(\psi_{-h}^{m} \circ \psi_{-h}^{n_{0}\left(x_{1}\right)} \circ g_{c_{i}} \circ \phi_{h}^{n_{0}\left(x_{1}\right)}\right)\left(x_{1}\right)=\psi_{-h}^{m}\left(\alpha\left(x_{1}\right)\right) \neq \alpha\left(x_{1}\right) .
\end{aligned}
$$

Surjectivity. If $y \in \mathcal{S}^{2}$ is a repelling fixed point, then $y=\alpha(y)$ (see definition of $\alpha$ ). Otherwise $y \in W_{\psi_{h}, c_{i}}^{\mathrm{s}}$ for some fixed point $c_{i}$ (attracting or saddle). Let $n_{0}=n_{0}(y)$ be such that

$y_{n_{0}-1}:=\psi_{h}^{n_{0}-1}(y) \in \operatorname{int}_{\text {rel }}\left(g\left(K_{c_{i}, 0}\right) \backslash g\left(K_{c_{i}, 1}\right)\right)$ and $y_{n_{0}}:=\psi_{h}^{n_{0}}(y) \in g\left(K_{c_{i}, 0}\right)$.

As $g$ is a conjugating homeomorphism,

$$
\left(\phi_{h}^{-1} \circ g^{-1} \circ \psi_{h}\right)\left(y_{n_{0}-1}\right)=g^{-1}\left(y_{n_{0}-1}\right) .
$$

Furthermore, since $\psi_{h}\left(y_{n_{0}-1}\right)=y_{n_{0}}$, we have

$$
\left(\phi_{h}^{-1} \circ g^{-1}\right)\left(y_{n_{0}}\right)=g^{-1}\left(y_{n_{0}-1}\right) \text {. }
$$

Set

$$
x_{n_{0}}:=g^{-1}\left(y_{n_{0}}\right) \quad \text { and } \quad x_{n_{0}-1}:=g^{-1}\left(y_{n_{0}-1}\right)=\phi_{h}^{-1}\left(x_{n_{0}}\right) .
$$

Since $g_{c_{i}}$ is a homeomorphism, $x_{n_{0}-1} \in\left(\right.$ int $\left._{\text {rel }} K_{c_{i}, 0} \backslash K_{c_{i}, 1}\right)$ and $x_{n_{0}} \in K_{c_{i}, 1}$. This means that for $x^{*}=\phi_{-h}^{n_{0}}\left(x_{n_{0}}\right)$ the natural number $n_{0}$ is the same as in the definition of $\alpha$, which implies that $y=\left(\psi_{h}^{-n_{0}} \circ g \circ \phi_{h}^{n_{0}}\right)\left(x^{*}\right)=\alpha\left(x^{*}\right)$.

\section{Continuity}

CASE 1: Continuity on the stable manifold of an attracting point. Let $x$ lie on the stable manifold of an attracting point $a_{i}$. There exists $n_{0}=n_{0}(x)$ such that

$$
\phi_{h}^{n_{0}}(x) \in K_{a_{i}, 1} \quad \text { and } \quad \phi_{h}^{n_{0}-1}(x) \in \operatorname{int}\left(K_{a_{i}, 0} \backslash K_{a_{i}, 1}\right) .
$$

If $\phi_{h}^{n_{0}}(x) \in \operatorname{int} K_{a_{i}, 1}$, then as $\operatorname{int} K_{a_{i}, 1}$ and $\operatorname{int}\left(K_{a_{i}, 0} \backslash K_{a_{i}, 1}\right)$ are open and the map $\phi(\cdot, t)$ is continuous, there exists a neighbourhood $U_{x}$ of $x$ such that

$$
\phi_{h}^{n_{0}}\left(U_{x}\right) \subset \operatorname{int} K_{a_{i}, 1} \quad \text { and } \quad \phi_{h}^{n_{0}-1}\left(U_{x}\right) \subset \operatorname{int}\left(K_{a_{i}, o} \backslash K_{a_{i}, 1}\right) .
$$

As $g_{i}, \psi_{-h}(\cdot, t)$ and $\phi_{h}(\cdot, t)$ are continuous, the map $\alpha=\psi_{-h}^{n_{0}} \circ g_{i} \circ \phi_{h}^{n_{0}}$ is continuous at $x$.

If $y:=\phi_{h}^{n_{0}}(x) \in \partial K_{a_{i}, 1}$, then every neighbourhood $U_{y}$ of $y$ intersects $\operatorname{int}\left(K_{a_{i}, 0} \backslash K_{a_{i}, 1}\right)$. Let $\left(y_{n}\right)_{n=1}^{\infty} \subset \operatorname{int}\left(K_{a_{i}, 0} \backslash K_{a_{i}, 1}\right)$ converge to $y$. Then there exists $N$ such that for every natural $n$ greater than $N, \phi_{h}\left(y_{n}\right) \in \operatorname{int} K_{a_{i}, 1}$. Indeed, suppose otherwise. Then there exists a subsequence $\left(y_{n_{k}}\right)$ such that $\phi_{h}\left(\left(y_{n_{k}}\right)_{k=1}^{\infty}\right) \not \subset \operatorname{int} K_{a_{i}, 1}$. However, $\phi_{h}(y) \in \operatorname{int} K_{a_{i}, 1}$ because the potential $E$ is constant on $\partial K_{a_{i}, 1}$ and decreases along a trajectory. This means that for every $\left.k, \varrho_{\mathcal{M}}\left(\phi_{h}(y), \phi_{h}\left(y_{n_{k}}\right)\right)>\inf \left\{\varrho_{\mathcal{M}}\left(\phi_{h}(y), w\right): w \in \partial K_{a_{i}, 1}\right)\right\}>0$. This is a contradiction because $\phi$ is continuous. 
We have demonstrated that for every $y \in \partial K_{a_{i}, 1}$ there exists a neighbourhood $U_{y}^{\star}$ with $\phi_{h}\left(U_{y}^{\star}\right) \subset K_{a_{i}, 1}$. The neighbourhood $U_{y}^{\star}$ has two disjoint components: the first one, $U_{y}^{\star 1}$, is included in int $K_{a_{1}, 1}$ whereas the second, $U_{y}^{\star 2}$, is not. The point $x$ is transformed by the map $\alpha$ in such a way that $U_{y}^{\star 1}$ is transformed by $g_{i}$, and $U_{y}^{\star 2}$ by $\psi_{-h} \circ g_{i} \circ \phi_{h}$. Afterwards $U_{y}^{\star 1} \cup U_{y}^{\star 2}$ is transformed by the map $\psi_{-h}^{n_{0}}$. But $g_{i}$ is a conjugating homeomorphism so

$$
\left(\psi_{-h} \circ g_{i} \circ \phi_{h}\right)\left(U_{y}^{\star 2}\right)=\left(\psi_{-h} \circ \psi_{h} \circ g_{i}\right)\left(U_{y}^{\star 2}\right)=g_{i}\left(U_{y}^{\star 2}\right)
$$

and therefore we can say that the whole neighbourhood $U_{y}^{\star}$ is transformed by $g_{i}$. The continuity of $\phi_{h}, g_{i}$ and $\psi_{-h}$ implies that $\alpha$ is continuous at $x$.

CASE 2: Continuity at repelling fixed points. There are two possibilities in this case: either

(i) the repelling point, say $p$, is in the closure of the stable manifold of a saddle point $q$, or

(ii) $p$ is in the closures of the stable manifolds of attracting points.

Suppose first that (i) holds.

STEP 1: Restriction to the stable manifold. Let $\left\{x_{k}\right\}_{k \in \mathbb{N}} \subset W_{\phi, q}^{\mathrm{s}}, p \in$ $\operatorname{cl} W_{\phi}^{\mathrm{s}}(q)$ and $\lim _{k \rightarrow \infty} x_{k}=p$.

Let $V_{q}$ be a neighbourhood of $q$ such that the homeomorphism $g_{h, q}$ conjugating $\phi_{h}$ and $\psi_{h}$ is defined on $V_{q}$. For sufficiently small $h$ and every natural $N$ almost all elements of the sequence $\left\{x_{k}\right\}_{k \in \mathbb{N}}$ have the following property:

$$
\phi_{h}^{n_{k}}\left(x_{k}\right) \in \operatorname{int} K_{q, 1}, \quad \phi_{h}^{n_{k}-1}\left(x_{k}\right) \in \operatorname{int}\left(K_{q, 0} \backslash K_{q, 1}\right), \quad n_{k}>N,
$$

which follows from Lemma 4.7 (for the definition of the sets $K_{q, 0}$ and $K_{q, 1}$, see Lemma 4.3). The same lemma also implies that there exists $r>0$ such that $y_{k}=\phi\left(x_{k}, n_{k} h\right) \notin B(q, r) \cap W_{\phi}^{\mathrm{s}}(q)=K_{q, 2}$ for all $k$. In other words, since the step on the manifold $\mathcal{M}$ is small, all the $y_{k}$ lie near $\partial K_{q, 1}$. Since $q$ is a fixed point of $g_{h, q}, g_{h, q}\left(V_{q}\right)$ is a neighbourhood of $q$ and $g_{h, q}\left(V_{q}\right) \cap W_{\phi_{h}}^{\mathrm{s}}(q) \subset$ $W_{\psi_{h}, q}^{\mathrm{s}}$ as $g_{h, q}$ locally conjugates $\phi_{h}$ and $\psi_{h}$. Since $p$ is also a repelling point of $\psi_{h}$, it lies in $W_{\psi_{h}}^{\mathrm{s}}(q)$ (by Corollary 4.11). As $g_{h, q}$ is a homeomorphism, $g_{q}\left(y_{k}\right) \notin g_{h, q}\left(K_{q, 2}\right)$ for every $k$. Let $W_{\psi_{h}}^{p, \mathrm{~s}}(q)$ be the maximal connected component of $W_{\psi_{h}}^{\mathrm{s}}(q) \backslash\{q\}$ containing $p$. Then, for every $\varepsilon>0$, there exists $N$ such that

$$
\psi_{-h}^{n}\left(W_{\psi_{h}}^{p, \mathrm{~s}}(q) \backslash K_{q, 2}\right) \subset B(p, \varepsilon) \quad \text { for all } n>N .
$$

Since almost all $n_{k}$ are greater than $N$ and every $y_{k}$ is in $W_{\psi_{h}}^{p, \mathrm{~s}}(q) \backslash K_{q, 2}$, almost all $\psi_{-h}^{n_{k}}\left(y_{k}\right)$ lie in $B(p, \varepsilon)$. This means that

$$
\lim _{k \rightarrow \infty} \psi_{-h}^{n_{k}}\left(g_{q}\left(y_{k}\right)\right)=p .
$$

Thus, we have shown that the map $\alpha$ restricted to $W_{\phi_{h}}^{\mathrm{s}}(q) \cup\{p\}$ is continuous at $p$. 
STEP 2: Continuity at the repelling point. Let $\varepsilon>0$. Since $\alpha$ is continuous on the stable manifold of every saddle point (see case 3 ), for every $x^{*}$ in such a manifold and $\varepsilon_{1}>0$, there exists $\delta_{1}>0$ such that if $x \in B\left(x^{*}, \delta_{1}\right)$ then $\alpha(x) \in B\left(x^{*}, \varepsilon_{1}\right)$. On the other hand, $p$ is a fixed point of $\alpha$, and $\alpha \mid W_{\phi}^{\mathrm{s}}(q)$ is continuous at $p$ (see step 1). Thus, for every $\varepsilon_{2}>0$ there exists $\delta_{2}>0$ such that if $x^{*} \in B\left(p, \delta_{2}\right)$, then $\alpha\left(x^{*}\right) \in B\left(p, \varepsilon_{2}\right)$. Let $\varepsilon_{1}$ and $\varepsilon_{2}$ be such that $\varepsilon_{1}+\varepsilon_{2}=\varepsilon$. Then the triangle inequality implies that if $x \in B\left(p, \delta_{1}+\delta_{2}\right)$ then $\alpha(x) \in B\left(p, \varepsilon_{1}+\varepsilon_{2}\right)$.

Suppose now that (ii) holds. Corollary 4.9 implies that there exists only one attracting singularity $a$ such that $p \in W_{\phi}^{\mathrm{s}}(a)$, whereas Lemma 4.8 implies that only one repelling point can lie in $\mathrm{cl} W_{\phi}^{\mathrm{s}}(a)$. This means that there exists a neighbourhood $V_{p}$ of $p$ such that

$$
\lim _{t \rightarrow \infty} \phi(x, t)=a
$$

for all $x \in V_{p} \backslash\{p\}$ and there exists a neighbourhood $V_{a}$ of $a$ such that

$$
\lim _{t \rightarrow \infty} \psi(y,-t)=p \quad \text { for all } y \in V_{a} \backslash\{a\} .
$$

We can now repeat the first step of subcase (i) to show that the map $\alpha$ is continuous at $p$.

CASE 3: Continuity on the stable manifold of a saddle point. Let $q_{i}$ be a saddle point. If the restriction $\phi_{h} \mid W_{\phi_{h}}^{\mathrm{s}}\left(q_{i}\right)$ is considered, then $q_{i}$ is an attracting singularity and, repeating the argument from case 1 , we have continuity of $\alpha$ in the relative topology on the stable manifold.

Thus, let $x_{0} \in W_{\phi_{h}}^{\mathrm{s}}\left(q_{i}\right)$ and $x \in U_{x_{0}} \backslash W_{\phi_{h}}^{\mathrm{s}}\left(q_{i}\right)$, where $U_{x_{0}}$ is a neighbourhood of $x_{0}$. Since there are no saddle-saddle connections, there exist attracting points $a_{j}, a_{k}$, not necessarily different, such that $W_{\phi_{h}}^{\mathrm{u}}\left(q_{i}\right)_{1} \subset W_{\phi_{h}}^{\mathrm{s}}\left(a_{j}\right)$ and $W_{\phi_{h}}^{\mathrm{u}}\left(q_{i}\right)_{2} \subset W_{\phi_{h}}^{\mathrm{s}}\left(a_{k}\right)$, where $W_{\phi_{h}}^{\mathrm{u}}\left(q_{i}\right)_{1}$ and $W_{\phi_{h}}^{\mathrm{u}}\left(q_{i}\right)_{2}$ are the connected components of the manifold $W_{\phi_{h}}^{\mathrm{u}}\left(q_{i}\right)$.

For every $x_{0} \in W_{\phi}^{\mathrm{s}}\left(q_{i}\right)$, each neighbourhood $V_{q_{i}}$ of $q_{i}$ and sufficiently small $h>0$, there exists $\delta_{1}>0$ and $n_{1}$ such that

$$
\phi_{h}^{n_{1}}\left(B\left(x, \delta_{1}\right)\right) \subset V_{q_{i}} .
$$

Suppose $V_{q_{i}} \cap W_{\phi}^{\mathrm{s}}\left(q_{i}\right)=\operatorname{int} K_{q_{1}, 1}$, and $h$ and $n_{1}$ are such that

$$
\phi_{h}^{n_{1}-1}\left(B\left(x_{0}, \delta_{1}\right) \cap W_{\phi}^{\mathrm{s}}\left(q_{i}\right)\right) \subset \operatorname{int}\left(K_{q_{i}, 0} \backslash K_{q_{i}, 1}\right) .
$$

Thus $x^{n_{1}}:=\phi_{h}^{n_{1}}(x)$ lies in the local stable manifold $W_{\phi, \text { loc }}^{\mathrm{s}}\left(q_{i}\right)$. Denote by $D_{\phi}^{\mathrm{u}}$ a disc transversal to $W_{\phi, \text { loc }}^{\mathrm{s}}\left(q_{i}\right)$, containing $x^{n_{0}}, x^{n_{1}}:=\phi_{h}^{n_{1}(x)}$ and embedded in $V_{q_{i}}$. According to the $\lambda$-lemma, for every $\delta_{2}>0$ and sufficiently small $h$, there exists $n_{2}$ such that

$$
D_{n_{2}}^{\mathrm{u}}:=\phi_{h}^{n_{2}}\left(D_{\phi}^{\mathrm{u}}\right)
$$


is $\delta_{2}$-close to $W_{\phi, l o c}^{\mathrm{u}}\left(q_{i}\right)$. Thus, for every $y \in D_{n_{2}}^{\mathrm{u}}$ there exists a point $\bar{y}$ in one of the connected components of $W_{\phi}^{\mathrm{u}}\left(q_{i}\right)$, for instance in $W_{\phi}^{\mathrm{u}}\left(q_{i}\right)_{1} \subset W_{\phi}^{\mathrm{s}}\left(a_{j}\right)$, such that

$$
\varrho_{\mathcal{M}}(y, \bar{y})<\delta_{2}
$$

For every neighbourhood $U_{q_{i}} \subset V_{q_{i}}$ of $q_{i}$ we can choose $x$ so close to $x_{0}$ that $y:=\phi_{h}^{n_{1}+n_{2}}(x) \in V_{q_{i}}$.

We will trace the behaviour of the point $y$ lying near the $W_{\phi}^{\mathrm{u}}\left(q_{i}\right)$ using a "spying point" $\bar{y}$ whose behaviour is known because it lies in $W_{\phi, \text { loc }}^{\mathrm{u}}\left(q_{i}\right)_{1} \subset$ $W_{\phi}^{\mathrm{s}}\left(a_{j}\right)$, where $a_{j}$ is an attracting point. Since $\bar{y}$ is in the attracting basin of $a_{j}$, there exists a neighbourhood $V_{\bar{y}} \subset W_{\phi}^{\mathrm{s}}\left(a_{j}\right)$. For every $\delta_{2}>0$, by the $\lambda$-lemma and continuity of $\phi_{h}$, we can choose $x$ so close to $x_{0}$ that $y \in B\left(\bar{y}, \delta_{2}\right)$. According to Lemmas 4.7 and 4.5 we can choose $n_{3}$ such that

$$
\phi_{h}^{n_{3}}(\bar{y})=: \overline{y^{n_{3}}} \in \operatorname{int} K_{a_{j}, 1}, \quad \phi_{h}^{n_{3}-1}(\bar{y}) \in \operatorname{int}\left(K_{a_{j}, 0} \backslash K_{a_{j}, 1}\right)
$$

and

$$
\phi_{h}^{n_{3}}(y)=: y^{n_{3}} \in \operatorname{int} K_{a_{j}, 1}, \quad \phi_{h}^{n_{3}-1}(y) \in \operatorname{int}\left(K_{a_{j}, 0} \backslash K_{a_{j}, 1}\right) .
$$

The map $\phi_{h}$ is continuous so, for every $\delta_{3}>0$, there exists $\delta_{2}>0$ such that if $\varrho_{\mathcal{M}}(\bar{y}, y)<\delta_{2}$, then $\varrho_{\mathcal{M}}\left(\overline{y^{n_{3}}}, y^{n_{3}}\right)<\delta_{3}$. Define

$$
\overline{z^{n_{3}}}:=\left(\psi_{-h}^{n_{3}} \circ g_{a_{j}}\right)\left(\overline{y^{n_{3}}}\right), \quad z^{n_{3}}:=\psi_{-h}^{n_{3}}\left(g_{a_{j}}\left(y^{n_{3}}\right)\right),
$$

where $g_{a_{j}}$ is a local conjugating homeomorphism constructed in Lemma 4.6. Then the definition of $g_{q_{i}, a_{j}}$ (see formula (12)) implies that

$$
g_{q_{i}}(\bar{y})=\overline{z^{n_{3}}},
$$

where $g_{q_{i}}$ is a local homeomorphism conjugating $\phi_{h}$ and $\psi_{h}$ in a neighbourhood of $q_{i}$. This follows from the definition since

$\overline{z^{n_{3}}}:=\left(\psi_{-h}^{n_{3}} \circ g_{q_{i}, a_{j}} \circ \phi_{-h}^{n_{3}}\right)(\bar{y})=\left(\psi_{-h}^{n_{3}} \circ\left(\psi_{h}^{n_{3}} \circ g_{q_{i}} \circ \phi_{-h}^{n_{3}}\right) \circ \phi_{h}^{n_{3}}\right)(\bar{y})=g_{q_{i}}(\bar{y})$.

Thus, for every neighbourhood $U_{q_{i}}$ of $q_{i}$, if $\varrho_{\mathcal{M}}\left(x, x_{0}\right)$ is sufficiently small, then the points $y, \bar{y}$ and $y_{0}:=g_{q_{i}}\left(\phi_{h}^{n_{1}+n_{2}}\left(x_{0}\right)\right)$ all lie in $U_{q_{i}}$. The continuity of the map $g_{q_{i}}$ and the equality $q_{q_{i}}\left(q_{i}\right)=q_{i}$ imply that for every $r>0$ there exists a neighbourhood $U_{q_{i}}$ such that $\overline{z^{n_{3}}}, g_{q_{i}}\left(y_{0}\right) \in B\left(q_{i}, r\right)$. Furthermore, $z^{n_{3}}$ is also in this ball by the continuity of $\psi_{-h}^{n_{3}} \circ g_{q_{i}, a_{j}} \circ \phi_{-h}^{n_{3}}$ and the equality $g_{q_{i}}(\bar{y})=\overline{z^{n_{3}}}$. The continuity of $\psi_{-h}$ assures that for every $r_{1}>0$ there exists a radius $r$ such that

$$
\varrho_{\mathcal{M}}\left(\psi_{-h}^{n_{2}}(y), \psi_{-h}^{n_{2}}\left(y_{0}\right)\right)<r_{1} .
$$

On the other hand $g_{q_{i}}\left(x_{0}^{n_{1}}\right)=\psi_{-h}^{n_{2}}\left(y_{0}\right)$ because 


$$
\begin{aligned}
\psi_{-h}^{n_{2}}\left(y_{0}\right) & =\left(\psi_{-h}^{n_{2}} \circ g_{q_{i}} \circ \phi_{h}^{n_{2}}\right)\left(x_{0}^{n_{1}}\right)=\left(\psi_{-h}^{n_{2}-1} \circ \psi_{-h} \circ g_{q_{i}} \circ \phi_{h} \circ \phi_{h}^{n_{2}-1}\right)\left(x_{0}^{n_{1}}\right) \\
& =\left(\psi_{-h}^{n_{2}-1} \circ \psi_{-h} \circ \psi_{h} \circ g_{q_{i}} \circ \phi_{h}^{n_{2}-1}\right)\left(x_{0}^{n_{1}}\right) \\
& =\left(\psi_{-h}^{n_{2}-1} \circ g_{q_{i}} \circ \phi_{h}^{n_{2}-1}\right)\left(x_{0}^{n_{1}}\right)=\ldots=\left(\psi_{-h} \circ g_{q_{i}} \circ \phi_{h}\right)\left(x_{0}^{n_{1}}\right) \\
& =\left(\psi_{-h} \circ \psi_{h} \circ g_{q_{i}}\right)\left(x_{0}^{n_{1}}\right)=g_{q_{i}}\left(x_{0}^{n_{1}}\right) .
\end{aligned}
$$

Recapitulating, for every $r_{1}>0$ we can choose the radius $r$ of the ball $B\left(q_{i}, r\right)$ such that

$$
\varrho_{\mathcal{M}}\left(\psi_{-h}^{n_{2}}(y), g_{q_{i}}\left(x_{0}^{n_{1}}\right)\right)<r_{1} .
$$

The continuity of $\psi_{h}$ implies that for every $\varepsilon>0$ there exists $r_{1}$ such that

$$
\varrho_{\mathcal{M}}\left(\psi_{-h}^{n_{1}}\left(\psi_{-h}^{n_{2}}(y)\right), \psi_{h}^{n_{1}}\left(g_{q_{i}}\left(x_{0}^{n_{1}}\right)\right)\right)=\varrho_{\mathcal{M}}\left(\alpha(x), \alpha\left(x_{0}\right)\right)<\varepsilon .
$$

This completes the proof.

Acknowledgments. I would like to thank Prof. Jerzy Ombach for many helpful discussions and suggestions.

\section{References}

[AD] F. Alouges and A. Debussche, On the qualitative behaviour of the orbits of a parabolic partial differential equation and its discretization in the neighbourhood of a hyperbolic fixed point, Numer. Funct. Anal. Optim. 12 (1991), 253-269.

[Bey1] W. J. Beyn, On invariant closed curves for one-step methods, Numer. Math. 51 (1987), 103-122.

[Bey2] -, On the numerical approximation of phase portraits near stationary points, SIAM J. Numer. Anal. 24 (1987), 1095-1113.

[BL] W. J. Beyn and J. Lorenz, Center manifolds of dynamical systems under discretizations, Numer. Funct. Anal. Optim. 9 (1987), 381-414.

[Bie] A. Bielecki, Gradient dynamical systems and learning process of layer artificial neural networks, PhD thesis, Faculty of Mathematics and Physics, Jagiellonian Univ., 1998 (in Polish).

[Fec1] M. Fečkan, Asymptotic behaviour of stable manifolds, Proc. Amer. Math. Soc. 111 (1991), 585-593.

[Fec2] -, Discretization in the method of averaging, ibid. 113 (1991), 1105-1113.

[Fec3] - , The relation between a flow and its discretization, Math. Slovaca 42 (1992), 123-127.

[Gar1] B. Garay, Discretization and some qualitative properties of ordinary differential equations about equilibria, Acta Math. Univ. Comenian. 62 (1993), 245-275.

[Gar2] -, Discretization and Morse-Smale dynamical systems on planar discs, ibid. 63 (1994), 25-38

[Gar3] -, On structural stability of ordinary differential equations with respect to discretization methods, J. Numer. Math. 4 (1996), 449-479.

[Gar4] - On $C^{j}$-closeness between the solution flow and its numerical approximation, J. Differential Equations Appl. 2 (1996), 67-86.

[Gar5] - The discretized flow on domains of attraction: a structural stability result, IMA J. Numer. Anal. 18 (1998), 77-90. 
[KL] P. E. Kloeden and J. Lorenz, Stable attracting sets in dynamical systems and their one-step discretizations, SIAM J. Numer. Anal. 23 (1986), 986-996.

[Kru] A. Krupowicz, Numerical Methods for Boundary Value Problems of Ordinary Differential Equations, PWN, Warszawa, 1986 (in Polish).

[Li] M. C. Li, Structural stability of Morse-Smale gradient-like flows under discretization, SIAM J. Math. Anal. 28 (1997), 381-388.

[Man] R. Mañé, A proof of $C^{1}$ stability conjecture, Publ. Math. IHES 66 (1988).

[MR] M. Mrozek and K. P. Rybakowski, Discretized ordinary differential equations and the Conley index, J. Dynam. Differential Equations 4 (1992), 57-63.

[PM] J. Palis and W. de Melo, Geometric Theory of Dynamical Systems, Springer, New York, 1982.

[Rob] J. Robbin, A structural stability theorem, Ann. of Math. 94 (1971), 447-493.

Institute of Computer Science

Jagiellonian University

Nawojki 11

30-072 Kraków, Poland

E-mail: uibielec@cyf-kr.edu.pl

Reçu par la Rédaction le 18.2.1999

Révisé le 2.8.1999 et le 8.12.1999 